\title{
Effect of the Mediterranean diet with and without weight loss on surrogate markers of cholesterol homeostasis in men with the metabolic syndrome
}

\author{
Caroline Richard $^{1}$, Patrick Couture ${ }^{1}$, Sophie Desroches ${ }^{2}$, Suzanne Benjannet ${ }^{3}$, Nabil G. Seidah ${ }^{3}$, \\ Alice H. Lichtenstein ${ }^{4}$ and Benoît Lamarche ${ }^{1 *}$ \\ ${ }^{1}$ Institute of Nutraceuticals and Functional Foods, Laval University, 2440, Boulevard Hochelaga, Quebec, QC, \\ Canada G1V OA6 \\ ${ }^{2} A x e$ de recherche en transfert des connaissances et évaluation des technologies et des modes d'interventions en santé, \\ CRCHUQ-HSFA, Quebec, QC, Canada \\ ${ }^{3}$ Institut de Recherches Cliniques de Montréal, Quebec, QC, Canada \\ ${ }^{4}$ Cardiovascular Nutrition Laboratory, Tufts University, Boston, MA, USA
}

(Received 19 January 2011 - Revised 17 May 2011 - Accepted 23 May 2011 - First published online 26 July 2011)

\section{Abstract}

The mechanisms implicated in the LDL-cholesterol (LDL-C)-lowering effects of the Mediterranean-type diet (MedDiet) are unknown. The present study assessed the impact of the MedDiet consumed under controlled feeding conditions, with and without weight loss, on surrogate markers of cholesterol absorption, synthesis and clearance using plasma phytosterols, lathosterol and proprotein convertase subtilisin/kexin-9 (PCSK9) concentrations, respectively, in men with the metabolic syndrome. The subjects' diet ( $n$ 19, 24-62 years) was first standardised to a baseline North American control diet ( 5 weeks) followed by a MedDiet ( 5 weeks), both under weight-maintaining isoenergetic feeding conditions. The participants then underwent a 20-week free-living energy restriction period (10 (SD 3 ) $\%$ reduction in body weight, $P<0 \cdot 01$ ), followed by the consumption of the MedDiet ( 5 weeks) under controlled isoenergetic feeding conditions. The LDL-C-lowering effect of the MedDiet in the absence of weight loss ( $-9.9 \%)$ was accompanied by significant reductions in plasma PCSK9 concentrations $(-11.7 \%, P<0.01)$ and in the phytosterol:cholesterol ratio $(-9.7 \%, P<0 \cdot 01)$ compared with the control diet. The addition of weight loss to the MedDiet had no further impact on plasma LDL-C concentrations and on these surrogate markers of LDL clearance and cholesterol absorption. The present results suggest that the MedDiet reduces plasma LDL-C concentrations primarily by increasing LDL clearance and reducing cholesterol absorption, with no synergistic effect of body weight loss in this process.

Key words: Mediterranean diet: Metabolic syndrome: Weight loss: Cholesterol homeostasis

The metabolic syndrome (MS) is a highly prevalent prothrombotic and pro-atherogenic condition characterised by abdominal obesity, hypertension, dyslipidaemia and insulin resistance ${ }^{(1)}$. Although raised plasma cholesterol concentration is not one of its typical features, the MS has also been associated with reduced intestinal cholesterol absorption and enhanced endogenous cholesterol synthesis ${ }^{(1,2)}$. While weight loss is recommended as the first step for the treatment of the MS, diet is being increasingly recognised as a key aetiological factor as well ${ }^{(1)}$. The Mediterranean-type diet (MedDiet) has been proposed as the optimal diet for the prevention of the $\mathrm{MS}^{(3)}$, having favourable effects on several risk factors including plasma LDL-cholesterol (LDL-C) concentrations $^{(4)}$. Little is known about the mechanisms that are implicated in the LDL-C-lowering effects of the MedDiet and how weight loss modulates these effects in the context of the MS. It has been suggested that the high phytosterol content of the MedDiet may on its own contribute to reduce plasma LDL-C concentrations by inhibiting intestinal absorption of cholesterol ${ }^{(5)}$. On the other hand, weight loss has been associated with reduced cholesterol synthesis, while having little or no effect on intestinal cholesterol absorption ${ }^{(6)}$.

Lathosterol is a cholesterol precursor and its plasma concentrations have been shown to reflect endogenous cholesterol synthesis $^{(7)}$. Plasma phytosterol concentrations, mostly campesterol and $\beta$-sitosterol, have been shown to be a valid surrogate marker of intestinal cholesterol absorption ${ }^{(7)}$. Moreover, studies have shown that markers of cholesterol synthesis and absorption may be stronger predictors of the risk of CVD than traditional plasma lipid concentrations ${ }^{(8)}$.

Abbreviations: LDL-C, LDL-cholesterol; MedDiet, Mediterranean diet; MS, metabolic syndrome; PCSK9, proprotein convertase subtilisin/kexin-9.

*Corresponding author: B. Lamarche, fax +1 4186565877 , email benoit.lamarche@inaf.ulaval.ca 
The proprotein convertase subtilisin/kexin-9 (PCSK9) also plays a key role in whole-body cholesterol homeostasis by regulating the intra-cellular degradation of the LDL receptor in the liver, small intestine and kidney ${ }^{(9)}$. Elevated plasma PCSK9 concentrations have been associated with a reduced clearance rate of $\mathrm{LDL}$ and hence with elevated plasma LDL-C concentrations ${ }^{(10)}$

The primary aim of the present study was to assess the impact of the MedDiet in the absence of weight change on surrogate markers of cholesterol absorption, synthesis and clearance using plasma phytosterols, lathosterol and PCSK9 concentrations, respectively, in male patients with the MS. The intervention was also designed to examine the extent to which body weight reduction modifies the impact of the MedDiet on these markers of cholesterol homeostasis.

\section{Subjects and methods}

The study design has already been described in detail previously $^{(4)}$. Briefly, men (18-65 years) from the Quebec City metropolitan area, who met the National Cholesterol Education Program Adult Treatment Panel (NCEP-ATP) III criteria for the $\mathrm{MS}^{(1)}$, were recruited for the study. All study procedures were approved by the Research Ethics Committee of Laval University (Quebec, QC, Canada). The present study was conducted according to the guidelines laid down in the Declaration of Helsinki, and written informed consent was obtained from all participants enrolled in the study. Men with a previous history of CVD or type 2 diabetes, on lipid-lowering or hypertension medication and smokers were excluded. Men had also to have a stable body weight for at least 6 months.

\section{Diets and study design}

Fig. 1 shows the study design ${ }^{(4)}$. The subjects' diet was first standardised to a baseline North American control diet that they consumed for 5 weeks under isoenergetic, weightmaintaining conditions. The participants then consumed the MedDiet for 5 weeks under isoenergetic, weight-maintaining conditions. This first part of the study in controlled feeding conditions (all foods were provided to the participants) allowed us to investigate the impact of the MedDiet per se in the absence of weight loss. The participants subsequently underwent a 20-week weight-loss period in free-living conditions during which they were given advice on how to create a $2092 \mathrm{~kJ}$ (500 kcal) deficit in their daily energy intake. This was achieved by recommending a reduction in portion size as well as by promoting low-energy density food choices in free-living conditions. Finally, the subjects consumed the MedDiet for the second time for 5 weeks under controlled feeding, weight-stabilising conditions. This allowed us to investigate the combined impact of the MedDiet and weight loss on markers of cholesterol homeostasis.

\section{Diets in the absence of weight loss}

The control diet was devised to reflect the current macronutrient intake averages in North American men ${ }^{(11)}$. The MedDiet was formulated to be concordant with the characteristics of the traditional Mediterranean eating pattern ${ }^{(12)}$. For the study, $7 \mathrm{~d}$ cyclic menus were developed and have already been presented ${ }^{(4)}$. The servings of various food categories consumed weekly during the control North American diet and the MedDiet for a $10460 \mathrm{~kJ}(2500 \mathrm{kcal})$ intake is presented in Table 1 . The control diet provided $34 \%$ of the energy as fat with $13 \%$ as SFA, $2 \%$ as trans-fatty acids, $13 \%$ as MUFA and $5 \%$ as PUFA; $48 \%$ of the energy as carbohydrates with a significant proportion of these coming from foods enriched in refined sugars; $17 \%$ of the energy as proteins and $2 \%$ of the energy as alcohol. The respective percentages of energy derived from lipids, carbohydrates, protein and alcohol in the MedDiet were 32, 50, 17 and 5\%, respectively. The MedDiet provided a MUFA:SFA ratio of 2.7 compared with a ratio of 1.0 in the control diet. The MedDiet also provided twice as much dietary fibre as the control diet, and virgin olive oil (commercially available) was the main source of fat in the diet. The experimental diets were formulated by using the Nutrition Data System software (version 4.03_31; Nutrition Coordinating Center, Minneapolis, MN, USA). Since the Nutrition Data System software does not provide amounts of phytosterols contained in foods, the phytosterol content of each diet was calculated using data from the United States Department of Agriculture National Nutrient Database for Standard Reference and from published tables ${ }^{(13-15)}$.

The control diet was first assigned using pre-determined levels of energy intake estimated using $3 \mathrm{~d}$ food records and

Time (weeks)
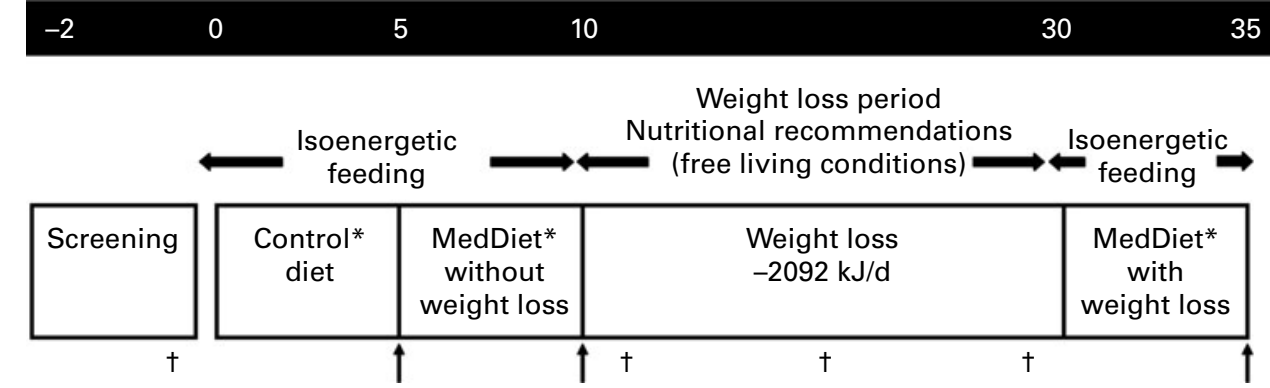

Fig. 1. Study design as shown in Richard et al. ${ }^{(4)}$. ${ }^{\star}$ Daily weighing and adjustment of energy intake (controlled feeding phases of the study). † Food journals and physical activity records $(3 \mathrm{~d})$. $\uparrow$ Blood samples taken for assessment of study outcomes. MedDiet, Mediterranean diet. 
Table 1. Servings of various food categories consumed weekly during the control North American diet and the Mediterranean diet (MedDiet) for a $10460 \mathrm{~kJ}(2500 \mathrm{kcal})$ menu

\begin{tabular}{lcc}
\hline Key foods & Control diet & MedDiet \\
\hline Whole-grain products (servings/d) & 1.2 & 5.4 \\
Fruits and vegetables (servings/d) & 6.6 & $16 \cdot 1$ \\
Legumes (servings/week) & 0.6 & 3.6 \\
Nuts (servings/d) & $0.5 \dagger$ & 0.9 \\
Cheese and yogurt (servings/d) & 1.8 & $2.0 \ddagger$ \\
Fish (servings/week) & 1.0 & $8 \cdot 8$ \\
Poultry (servings/d) & 1.0 & 0.9 \\
Eggs (servings/week) & 2.6 & $2 \cdot 2$ \\
Sweets (servings/week) & 13.0 & 2.0 \\
Red meat (servings/d) & 1.9 & 0.2 \\
Red wine (servings/d) & 1.0 & 2.6 \\
Olive oil (g/week) & 4.5 & 302.8 \\
\hline
\end{tabular}

* Olive oil, g/week; portion size for whole-grain products, $125 \mathrm{ml}$ (brown rice, brown pasta, bulgur and couscous), one bread piece or $30 \mathrm{~g}$ cereal; for fruits and vegetables, $125 \mathrm{ml}$; for legumes, $175 \mathrm{ml}$; for nuts, $30 \mathrm{~g}$; for fish, poultry and red meat, $75 \mathrm{~g}$; for eggs, $100 \mathrm{~g}$; for dairy products, $50 \mathrm{~g}$ cheese, $175 \mathrm{~g}$ yogurt and $250 \mathrm{ml}$ milk; for one glass of red wine, $75 \mathrm{ml}$.

$\dagger$ Mostly peanut butter on the control diet.

‡ Low-fat cheese and yogurt on the MedDiet.

revised according to body weight fluctuations measured daily during the isoenergetic phases of the study. All meals, foods and beverages including red wine were provided to participants at the clinical investigation unit during the isoenergetic phases of the study, thereby optimising control for energy and macronutrient intake. The men were instructed to only consume the meals provided and to maintain their usual levels of physical activity during all isoenergetic phases of the study ( 15 weeks). A checklist was provided to all participants to identify the prescribed foods that had not been consumed when eating outside of the clinical investigation unit (mostly dinners and breakfasts) or had been consumed in addition to the formulated diets. Information on physical activity was collected using a daily checklist.

\section{Weight loss period and stabilisation on the Mediterranean diet}

After the first isoenergetic phase, the participants received instructions on how to reduce their usual energy intake by $2092 \mathrm{~kJ}(500 \mathrm{kcal}) / \mathrm{d}$, in order to generate a minimum of $5-10 \%$ reduction in body weight over a period of 20 weeks. To be consistent with our second objective, only participants who had lost at least $5 \%$ of their body weight were eligible for the last isoenergetic phase of the study during which they consumed the MedDiet for 5 weeks again under isoenergetic, weight-stabilising conditions, with all foods provided to them as in the first isoenergetic phase.

\section{Laboratory measurements}

The lipid profile and lipoprotein-lipid concentrations as well as insulin and glucose levels were assessed after a $12 \mathrm{~h}$ fast at the end of each experimental isoenergetic diet according to previously described methods ${ }^{(4)}$. Anthropometric measures were assessed according to standardised procedures ${ }^{(16)}$. Plasma concentrations of lathosterol, $\beta$-sitosterol and campesterol were quantified using a GC method similar to the one described previously ${ }^{(8)}$. Total plasma phytosterol concentrations were calculated as the sum of plasma campesterol and $\beta$-sitosterol concentrations. All values have been expressed as ratios relative to plasma total cholesterol concentrations $\left(\times 10^{2} \mu \mathrm{mol} / \mathrm{mmol} \text { of cholesterol }\right)^{(7)}$. Plasma PCSK9 concentrations were measured in fasting plasma at the end of each experimental isoenergetic diet by an in-house ELISA as described in Dubuc et al. ${ }^{(17)}$.

\section{Statistical analysis}

Data are reported as means and standard deviations and percentage of changes from the control diet unless stated otherwise. The main analysis compared the values of each outcome measured at the end of the control diet, MedDiet without weight loss and MedDiet with weight loss. Data were analysed using the PROC MIXED procedure for repeated measures in SAS (version 9.2; SAS Institute, Inc., Cary, NC, USA). The Tukey adjustment in the MIXED model was used to account for multiple comparisons of the three diets a posteriori. The extent to which initial values (on the control diet) modified the change in risk factors in response to the MedDiet with and without weight loss was also investigated using appropriate interaction terms in the MIXED model. When the interaction terms were found to be significant, the subgroups were created using the median value for the selected variable measured after the control diet to better illustrate this interaction. Variables with a skewed distribution were $\log _{10}$ transformed before statistical analysis. Differences at $P \leq 0.05$ (two-sided) were considered significant.

\section{Results}

Of the twenty-nine men recruited in the study, two subjects dropped out and eight subjects were excluded because of non-compliance with the study protocol and requirements ${ }^{(4)}$. Of these subjects, seven did not achieve the $5 \%$ body weight loss prescription defined a priori. The mean weight loss in these seven subjects was $1 \cdot 2$ (SD 1.7) $\%$ and was therefore not sufficient for including these subjects in the last phase of the study. The characteristics at screening of the nineteen participants included in the analyses are shown in Table 2 . The overall compliance with the pre-determined diets during feeding in the isoenergetic phases of the study calculated from the food checklist in these subjects was 97.9 (SD $1 \cdot 8) \%$.

As shown previously, the MedDiet in the absence of weight loss significantly reduced plasma total cholesterol $(-7.8 \%)$, LDL-C (-9.9\%) and LDL-apo B (-10.4\%) compared with the control diet (all $P<0 \cdot 01$, Table 3$)^{(4)}$. Although the estimated intake in dietary phytosterol increased by 288.0 (SD 48.5$) \mathrm{mg} / \mathrm{d}$ compared with the control diet, consumption of the MedDiet in the absence of weight loss led to a significant $9.7 \%$ reduction in the plasma phytosterol:cholesterol ratio $(P=0 \cdot 007)$. There was no significant correlation between changes in phytosterol intake and changes in the plasma phytosterol:cholesterol ratio (not shown). Finally, plasma PCSK9 
Table 2. Physical characteristics and plasma lipid profile at screening of the nineteen male subjects included in analyses (Mean values and standard deviations)

\begin{tabular}{|c|c|c|}
\hline Variable & Mean & SD \\
\hline Age (years) & $50 \cdot 8$ & $10 \cdot 8$ \\
\hline Weight (kg) & 99.5 & $19 \cdot 2$ \\
\hline BMI $\left(\mathrm{kg} / \mathrm{m}^{2}\right)$ & 33.4 & 5.5 \\
\hline Waist circumference $(\mathrm{cm})$ & 112.5 & $11 \cdot 7$ \\
\hline Total-C (mmol/l) & 5.46 & 1.06 \\
\hline LDL-C (mmol/l) & 3.53 & 0.97 \\
\hline $\mathrm{HDL}-\mathrm{C}(\mathrm{mmol} / \mathrm{l})$ & 1.00 & 0.26 \\
\hline TAG (mmol/l) & 2.02 & 0.78 \\
\hline MS (\%) & \multicolumn{2}{|c|}{100} \\
\hline
\end{tabular}

C, cholesterol; MS, metabolic syndrome.

concentrations were reduced by $11.7 \%$ after the MedDiet in the absence of weight loss compared with the control diet $(P=0.004)$. Changes in plasma PCSK9 concentrations after the MedDiet in the absence of weight loss correlated significantly with concurrent reduction in plasma LDL-C $(r \quad 0 \cdot 40$, $P=0.088)$ and in LDL-apoB concentrations ( $r 0.51, P=0.026)$.

The 20-week energy restriction phase and further stabilisation on the MedDiet for 5 weeks reduced the body weight by 10.2 (SD 2.9) \% and waist circumference by $8.6(\mathrm{SD} 3.3) \mathrm{cm}$ compared with the values measured after the control diet $(P<0 \cdot 001)^{(4)}$. Combining weight loss with the MedDiet had no further impact on plasma total-cholesterol, LDL-C and PCSK9 concentrations. However, combining weight loss with the MedDiet significantly reduced the plasma lathosterol: cholesterol ratio compared with the MedDiet in the absence of weight loss $(-18.2 \%, P=0.004)$ and the control diet $(-17.5 \%, P=0.007)$. The magnitude of the reduction in the plasma lathosterol:cholesterol ratio on the MedDiet with weight loss from values on the control diet was positively correlated with parallel changes in body weight, TAG and insulin $(0.46<r<0.52$, all $P<0.05)$. Finally, combining weight loss to the MedDiet tended to increase the plasma phytosterol:cholesterol ratio compared with the values on the MedDiet in the absence of weight loss $(8.3 \%, P=0.075)$.

Fig. 2 illustrates how baseline plasma PCSK9 concentrations on the control diet modulated both the PCSK9 and the LDL-apoB response to the MedDiet with and without weight loss. The MedDiet, irrespective of weight loss, was associated with a significant reduction in plasma PCSK9 and LDL-apoB concentrations only in men with arbitrarily higher plasma PCSK9 concentrations at baseline $(P<0.04$ for both interactions).

\section{Discussion}

Elevated plasma LDL-C concentrations are typically not considered a central feature of the MS. However, studies show that insulin resistance and the MS are associated with a perturbed cholesterol homeostasis encompassing reduced intestinal cholesterol absorption and increased whole-body cholesterol synthesis ${ }^{(2)}$. We believe that this is the first nutritional intervention documenting the impact of the MedDiet in the absence and presence of weight loss on surrogate markers

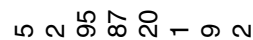
कํ்

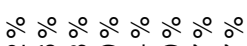
ํㅜㄴ - ó ó 응 ó 

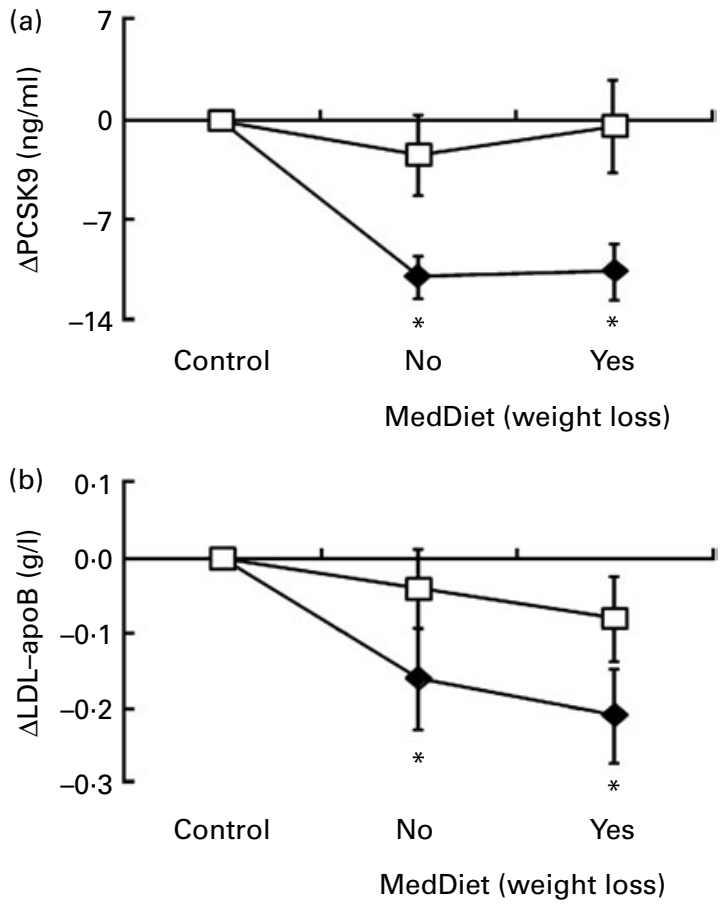

Fig. 2. (a) Proprotein convertase subtilisin/kexin-9 (PCSK9) and (b) LDLapoB response to the Mediterranean diet (MedDiet) with and without weight loss according to baseline values of PCSK9. The $P$ value for the interaction was obtained from the corresponding interactions terms in the MIXED model, using each variable as a continuous variable. The median value of PCSK9 $(57.8 \mathrm{ng} / \mathrm{ml})$ measured after the control diet was used to categorise individuals with high or low levels at baseline and to present this interaction graphically. ${ }^{*}$ Values were significantly different from those of control diet $(P<0.05)$. Baseline PCSK9: $-\downarrow-$, high; $-\square-$, low. (a) $P_{\text {interaction }}<0.001$ and (b) $P_{\text {interaction }}=0.037$

of cholesterol homeostasis in men with the MS. To the best of our knowledge, this is the first study of the MedDiet undertaken under tightly controlled feeding conditions as all previous studies are either epidemiological in nature or of interventions with dietary recommendations.

\section{Mediterranean diet in the absence of weight loss}

Our data suggest that part of the cholesterol-lowering properties of the MedDiet may be attributed to the reduced intestinal cholesterol absorption. The $8-10 \%$ reduction in plasma total cholesterol and LDL-C concentrations observed with the MedDiet in the present study ${ }^{(4)}$ is comparable in magnitude with data from previous studies ${ }^{(18)}$. Escurriol et al. ${ }^{(19)}$ have suggested that even small increases in the intake of phytosterols from traditional Mediterranean foods may play a role in the cholesterol-lowering effect of the MedDiet. $\beta$-Sitosterol, the most abundant phytosterol in the MedDiet, has been shown to reduce the expression of Niemann-Pick C1-like $1^{(20)}$, the major intestinal cholesterol transporter, and this mechanism may indeed be evoked to explain the reduction in cholesterol absorption with the MedDiet in the absence of weight loss. The MedDiet in the present study only provided an extra $288 \mathrm{mg} / \mathrm{d}$ of dietary phytosterols compared with the control diet, and it is thought that several hundred fold increase in the dietary load of phytosterols only doubles the plasma $\beta$-sitosterol concentrations in humans ${ }^{(21)}$. We also stress that total plasma phytosterol concentrations were reduced with the MedDiet in the absence of weight loss despite this small increase in dietary phytosterol intake, and there was no correlation between variations in plasma phytosterol concentrations and dietary intake. Although some concerns have been raised regarding how variations in dietary phytosterol may affect the interpretation of results based on plasma phytosterol concentrations as surrogate markers of cholesterol absorption, we argue that the MedDiet-induced variation in plasma phytosterol concentrations are still likely to reflect whole diet effects rather than changes in dietary phytosterol intake per se. On the other hand, increased dietary intake of MUFA, PUFA, fish oils and fibres have been associated with reduced cholesterol absorption $^{(22)}$. Therefore, the higher intake of these nutrients may also partly explain the reduction in cholesterol absorption with the MedDiet.

PCSK9 plays a determining role in modulating the intracellular degradation of the LDL receptor ${ }^{(9)}$. PCSK9 knockout mice have reduced plasma total cholesterol concentrations compared with wild-type mice due to an increased LDL receptor activity that accelerates clearance of LDL particles ${ }^{(23)}$. The MedDiet in the absence of weight loss led to a significant reduction in plasma PCSK9 concentrations compared with the control diet, and the magnitude of the PCSK9 reduction was significantly correlated with the concurrent reduction in plasma LDL-apoB concentrations. This correlation remained significant even after adjustment for concurrent variations in plasma phytosterol concentrations with the MedDiet (not shown). This suggests that a significant proportion of the reduction in plasma LDL-C and LDL-apoB concentrations observed with the MedDiet in the absence of weight loss may be due to an enhanced uptake of LDL from the bloodstream by various tissues including the liver, independent of the apparent reduction in cholesterol absorption.

The MedDiet in the absence of weight loss had no effect on cholesterol synthesis as estimated by plasma lathosterol concentrations. Reduction in cholesterol absorption is generally counterbalanced by increased cholesterol synthesis ${ }^{(6)}$. The 3-hydroxy-3methylglutaryl-CoA reductase, the rate-limiting enzyme in the cholesterol synthesis pathway, seems to be mostly regulated by intracellular cholesterol concentrations through negative feedback mechanisms ${ }^{(24)}$. We hypothesise that an increased uptake of LDL particles by the liver, thought to occur because of a reduced PCSK9 expression, may have contributed to maintaining the intracellular cholesterol pool that may have otherwise been reduced due to lower cholesterol absorption following the consumption of the MedDiet in the absence of weight loss ${ }^{(6,24)}$. Replacing dietary SFA with MUFA has been shown to increase LDL clearance, which could be partly and indirectly responsible for the neutral effect of the MedDiet on cholesterol synthesis ${ }^{(25)}$. Among other dietary factors known to affect endogenous cholesterol synthesis, plant sterols and PUFA have been associated with increased cholesterol synthesis ${ }^{(26)}$. 


\section{Mediterranean diet in the presence of weight loss}

The study also allowed us to specifically investigate the combined effect of weight loss and the MedDiet on surrogate markers of cholesterol homeostasis. Total plasma phytosterol concentrations on the MedDiet after weight loss tended to be higher than values before weight loss and were back to values measured after the control diet. The MedDiet combined with weight loss led to a significant reduction in the estimated cholesterol synthesis compared with the control diet and the MedDiet in the absence of weight loss, and this is consistent with studies having shown that weight loss frequently leads to reduction in cholesterol synthesis ${ }^{(6)}$. Insulin resistance, on the other hand, has been associated with an increased endogenous cholesterol synthesis ${ }^{(27)}$. This is consistent with our data showing that the reduction in cholesterol synthesis on the MedDiet with weight loss correlated with the reductions in body weight and in plasma TAG and insulin concentrations, which altogether predicts reduced secretion of apoB-containing lipoproteins by the liver. Such conclusions must be made with caution, however, without any confirmatory kinetic data from stable isotope studies. Cholesterol synthesis was also inversely correlated with cholesterol absorption after the MedDiet with weight loss (not shown). Based on these observations, we suggest that estimated cholesterol absorption may have been up-regulated after the MedDiet with weight loss $v$. before weight loss to compensate for the weight loss-induced reduction in endogenous cholesterol synthesis, thus attenuating any further change in plasma cholesterol concentrations.

Finally, previous studies have shown that weight loss on a low-fat diet is associated with an increased LDL clearance in patients with the $\mathrm{MS}^{(28)}$. As indicated earlier, the MedDiet in the absence of weight loss reduced plasma PCSK9 concentrations and the addition of weight loss had no further impact on this surrogate marker of LDL clearance. This suggests that weight loss per se may have no influence on estimated LDL clearance when changes have already occurred through significant dietary modifications with the MedDiet. Indeed, comparing the values before and after weight loss measured in both instances after controlled feeding of the MedDiet provides information on the effect of weight loss per se on cholesterol homeostasis markers since the dietary pattern and hence nutrient intake are unchanged. Taken together, the present results suggest that change in estimated LDL clearance with diet may be a more important determinant of the plasma LDL-C and apoB response than change in cholesterol synthesis.

Inter-individual variation in the cholesterol response to the MedDiet was quite important, and our data revealed that baseline PCSK9 concentrations were a significant determinant of this variation. Specifically, patients with a low estimated LDL clearance (reflected by high plasma PCSK9 levels) showed a greater reduction in plasma LDL-apoB concentrations on the MedDiet with and without weight loss than patients with an elevated clearance to start with. Although the significance of varying plasma PCSK9 in plasma is not known, the present results suggest that plasma PCSK9 may represent an interesting and novel biomarker predicting the LDL response to the MedDiet in men with MS

In summary, the reduction in plasma LDL-C concentrations with the MedDiet even in the absence of weight loss appears to be primarily due to an increased LDL clearance combined with reduced intestinal cholesterol absorption, rather than to any change in endogenous cholesterol synthesis. Adding weight loss to the MedDiet has no further effect on plasma cholesterol concentrations, most probably because there is no further change in LDL clearance and the reduction in cholesterol synthesis with weight loss may have been counterbalanced by a reciprocal elevation in cholesterol absorption. However, these conclusions have to be interpreted with caution without the kinetic data on apoB-containing lipoproteins and cholesterol with stable isotopes.

\section{Acknowledgements}

B. L., P. C., A. H. L. and S. D. have designed and obtained funding for the present study. P. C. is a recipient of a scholarship from the FRSQ. N. G. S. is a recipient of Canada Research Chair in Precursor Proteolysis (no. RCHS0164). C. R. contributed to the coordination of the clinical study, analysed the data and wrote the manuscript, which was reviewed critically by all authors. B. L. is a Canada Research Chair in Nutrition and Cardiovascular Health. S. D. is a FRSQ Junior 1 scholar. We are grateful to the subjects for their excellent collaboration and to the dedicated staff of the Institute on Nutraceutical and Functional Food. The present study was supported by an operating grant from the Canadian Institutes for Health Research (MOP-68866, MOP-102741). Provigo/Loblaws donated the foods used in the present study. The authors report no conflict of interest in relation to the present study.

\section{References}

1. Grundy SM, Cleeman JI, Daniels SR, et al. (2005) Diagnosis and management of the metabolic syndrome: an American Heart Association/National Heart, Lung, and Blood Institute Scientific Statement. Circulation 112, 2735-2752.

2. Gylling H, Hallikainen M, Kolehmainen M, et al. (2007) Cholesterol synthesis prevails over absorption in metabolic syndrome. Transl Res 149, 310-316.

3. Meydani M (2005) A Mediterranean-style diet and metabolic syndrome. Nutr Rev 63, 312-314.

4. Richard C, Couture P, Desroches S, et al. (2010) Effect of the Mediterranean diet with and without weight loss on cardiovascular risk factors in men with the metabolic syndrome. Nutr Metab Cardiovasc Dis (Epublication ahead of print version 27 May 2010).

5. Rudkowska I \& Jones PJ (2007) Functional foods for the prevention and treatment of cardiovascular diseases: cholesterol and beyond. Expert Rev Cardiovasc Ther 5, 477-490.

6. Santosa S, Varady KA, AbuMweis S, et al. (2007) Physiological and therapeutic factors affecting cholesterol metabolism: does a reciprocal relationship between cholesterol absorption and synthesis really exist? Life Sci $\mathbf{8 0}, 505-514$.

7. Simonen P, Gylling H \& Miettinen TA (2008) The validity of serum squalene and non-cholesterol sterols as surrogate markers of cholesterol synthesis and absorption in type 2 diabetes. Atherosclerosis 197, 883-888. 
8. Matthan NR, Giovanni A, Schaefer EJ, et al. (2003) Impact of simvastatin, niacin, and/or antioxidants on cholesterol metabolism in CAD patients with low HDL. J Lipid Res $\mathbf{4 4}$, 800-806.

9. Seidah NG (2009) PCSK9 as a therapeutic target of dyslipidemia. Expert Opin Ther Targets 13, 19-28.

10. Chan DC, Lambert G, Barrett PH, et al. (2009) Plasma proprotein convertase subtilisin/kexin type 9: a marker of LDL apolipoprotein B-100 catabolism? Clin Chem $\mathbf{5 5}$, 2049-2052.

11. Gray-Donald K, Jacobs-Starkey L \& Johnson-Down L (2000) Food habits of Canadians: reduction in fat intake over a generation. Can J Public Health 91, 381-385.

12. Matalas A-L, Zampelas A, Stavrinos V, et al. (editors) (2001) The Mediterranean Diet: Constituents and Health Promotion. Boca Raton, FL: CRC Press.

13. Kritchevsky D (1997) Phytosterols. Adv Exp Med Biol 427, $235-243$.

14. Normen L, Johnsson M, Andersson H, et al. (1999) Plant sterols in vegetables and fruits commonly consumed in Sweden. Eur J Nutr 38, 84-89.

15. Normen LBS \& Johnsson M (2002) The phytosterol content of some cereal foods commonly consumed in Sweden and in the Netherlands. J Food Compos Anal 15, 693-704.

16. Lohman TG, Roche AF \& Martorell R (1988) Anthropometric Standardization Reference Manual. Champaign, IL: Human Kinetics Books.

17. Dubuc G, Tremblay M, Pare G, et al. (2010) A new method for measurement of total plasma PCSK9: clinical applications. J Lipid Res 51, 140-149.

18. Jula A, Marniemi J, Huupponen R, et al. (2002) Effects of diet and simvastatin on serum lipids, insulin, and antioxidants in hypercholesterolemic men: a randomized controlled trial. JAMA 287, 598-605.
19. Escurriol V, Cofan M, Serra M, et al. (2009) Serum sterol responses to increasing plant sterol intake from natural foods in the Mediterranean diet. Eur J Nutr 48, 373-382.

20. Jesch ED, Seo JM, Carr TP, et al. (2009) Sitosterol reduces messenger RNA and protein expression levels of NiemannPick C1-like 1 in FHs 74 Int cells. Nutr Res 29, 859-866.

21. Miettinen TA, Tilvis RS \& Kesaniemi YA (1990) Serum plant sterols and cholesterol precursors reflect cholesterol absorption and synthesis in volunteers of a randomly selected male population. Am J Epidemiol 131, 20-31.

22. Wang DQ (2007) Regulation of intestinal cholesterol absorption. Annu Rev Physiol 69, 221-248.

23. Rashid S, Curtis DE, Garuti R, et al. (2005) Decreased plasma cholesterol and hypersensitivity to statins in mice lacking Pcsk9. Proc Natl Acad Sci U S A 102, 5374-5379.

24. Liscurn L, Dennis EV \& Jean EV (2002) Cholesterol biosynthesis. In New Comprehensive Biochemistry, chapter 15 pp. 409-431. Amsterdam, The Netherlands: Elsevier.

25. Gill JM, Brown JC, Caslake MJ, et al. (2003) Effects of dietary monounsaturated fatty acids on lipoprotein concentrations, compositions, and subfraction distributions and on VLDL apolipoprotein B kinetics: dose-dependent effects on LDL. Am J Clin Nutr 78, 47-56.

26. Jones PJ (1997) Regulation of cholesterol biosynthesis by diet in humans. Am J Clin Nutr 66, 438-446.

27. Pihlajamaki J, Gylling H, Miettinen TA, et al. (2004) Insulin resistance is associated with increased cholesterol synthesis and decreased cholesterol absorption in normoglycemic men. J Lipid Res 45, 507-512.

28. Ng TW, Watts GF, Barrett PH, et al. (2007) Effect of weight loss on LDL and HDL kinetics in the metabolic syndrome: associations with changes in plasma retinol-binding protein-4 and adiponectin levels. Diabetes Care 30, $2945-2950$. 